\title{
An Empirical Analysis of the Band Characteristics under the Influence of RMB Exchange Rate System Reform
}

\author{
Xiaoling Song, Xin Yue* \\ Business School, Beijing Language and Culture University, Beijing, 100083, China \\ *corresponding author
}

Keywords: RMB exchange rate, Exchange rate regime reform, GARCH Model.

\begin{abstract}
Based on the long-term trend of the exchange rate between the US dollar and the RMB, the paper establishes the GARCH model in five phases of the RMB exchange rate series, and empirically analyzes the band characteristics of the RMB exchange rate. The empirical results show that the expansion of exchange rate volatility limits increases the sensitivity of the exchange rate series, and the memory is weakened. Although the volatility is significantly increased, the effect of stabilizing the exchange rate is enhanced. The central bank should further deepen the reform of the renminbi exchange rate, combine the formation of the exchange rate mechanism and the adjustment of volatility limits to bring the market efficiency of the renminbi exchange rate into play.
\end{abstract}

\section{Introduction}

With the development of economic globalization, the RMB exchange rate reform continues to deepen. What kind of influence does exchange rate reform have on RMB exchange rate fluctuations? Based on the previous studies, this paper constructs the GARCH model and studies the fluctuation characteristics of the RMB exchange rate series under different exchange rate policies.

In the study of exchange rate sequences, the GARCH family model is widely used. Wang Jiani and Li Wenhao (2005) used the GARCH model to analyze the exchange rate of Euro, Japanese yen, British pound, and Australian dollar against the U.S. dollar from 1999 to 2004, and forecasted the volatility rate, resulting in the GARCH(1,1) model. To better predict the exchange rate of the yen against the US dollar, the ARCH(1) model can better predict the fluctuation of the exchange rate of the pound to the U.S. dollar. In the study of the RMB exchange rate sequence, scholars have generally verified the applicability of the GARCH $(1,1)$ model in the prediction of RMB exchange rate volatility. Luo Wei and Wu Jianhong (2009) used the GARCH model to analyze the daily data of the exchange rate of the US dollar against the RMB before and after the reform of the RMB exchange rate formation mechanism in 2005. This confirmed that the ARCH effect actually exists in the foreign exchange market in China, and found that the GARCH model can better fit the RMB exchange rate data after the exchange rate reform. Zhang Heng and Wu Ke (2015) analyzed the daily data of the exchange rate of the US dollar against the US dollar from the exchange rate reform on July 21, 2005 to February 28, 2015, and established GARCH $(1,1)$ in three phases. The model empirically examines the dynamic characteristics of the RMB exchange rate band and finds that the RMB exchange rate as a whole fluctuates drastically and is characterized by persistence, agglomeration, and memory. Zhang Jian (2018) used the time series threshold generalized autoregressive conditional heteroscedastic model (Threshold GARCH) to analyze the impact of the previous RMB exchange rate volatility adjustment on the RMB exchange rate volatility mechanism since the reform of the RMB exchange rate formation mechanism on July 21, 2005. The positive influence of the heteroscedasticity of the first-period condition is stronger than that of the lag disturbance item.

Summarizing the findings of the above scholars, the GARCH $(1,1)$ model can fit the fluctuation series of the RMB exchange rate. However, the RMB exchange rate fluctuations before and after the reform of the RMB exchange rate system show a large difference. Drawing lessons from previous model experiences, this paper analyses the fluctuation characteristics of the RMB exchange rate 
before and after the reform of the exchange rate system and further analyzes the impact of the exchange rate reform policy on exchange rate fluctuations. The exchange rate system reform proposed relevant policy recommendations.

\section{RMB Exchange Rate Reform Process}

On January 1, 1994, China adjusted the dual exchange rate system to a single exchange rate system and implemented a single, managed, floating exchange rate system based on market supply and demand. The official exchange rate of RMB 5.8 yuan was adjusted to a market exchange rate of US\$ 8.7 yuan. On July 21, 2005, China changed its exchange rate system linked to the US dollar over ten years to refer to a basket of currencies for adjustment and a single, managed floating exchange rate system. The People's Bank of China announces the closing price of the exchange rate of the US dollar and other currencies on the inter-bank foreign exchange market on the day after the close of the market on each working day, as the middle price of the RMB exchange rate on the next business day, and the daily exchange rate of the dollar on the inter-bank foreign exchange market. The trading price of the RMB must be within a range of $0.3 \%$ above and below the midpoint of the U.S. dollar exchange rate announced by the People's Bank of China. In August 2008, when the financial crisis broke out, the reform of the RMB exchange rate formation mechanism once stagnated and returned to the peg to the dollar. On June 19, 2010, the central bank resumed the process of RMB exchange rate reform. The exchange rate between the US dollar and the RMB in the interbank foreign exchange market must be within $0.5 \%$ of the central parity rate of the US dollar announced by the People's Bank of China. Starting from March 17, 2014, the daily exchange rate of RMB against the US dollar in the inter-bank spot foreign exchange market was adjusted to a volatility of $2 \%$ above and below. On August 11, 2015, when setting the mid-point price of RMB, we first took reference to the supply and demand situation of the previous day's closing exchange rate; at the same time, with reference to a basket of currency exchange rate changes, the volatility limit was adjusted to $3 \%$.

\section{GARCH Model}

Engle proposed an autoregressive conditional heteroscedastic model (ARCH) in 1982, but for financial sequences using daily or weekly data, the variance of $\mu_{t}$ often depends on the amount of change before many moments, which requires estimating many parameters. In this regard, Bollerslev (1986) proposed a generalized autoregressive conditional heteroscedastic model (GARCH). Apart from the common regression model, the GARCH model further models the error variance. The basic idea is to use one or two $\sigma_{t}^{2}$ lag values instead of $\mu_{t}{ }^{2}$ lag values, then the equation is greatly simplified. This article uses the GARCH $(1,1)$ model, which is an autoregressive term with a lag order of 1 and a residual squared item with a lag order of 1 . The expressions of the model are:

$$
\begin{gathered}
\mathrm{yt}=\lambda \mathrm{xt}+\mu \mathrm{t} \\
\sigma \mathrm{t} 2=\omega+\alpha \mu \mathrm{t}-12+\beta \sigma \mathrm{t}-12
\end{gathered}
$$

Among them, the formula (1) gives an exogenous function (mean equation) with an error term. $\lambda$ is the coefficient of the variable and $\mathrm{x}_{\mathrm{t}}$ is the exogenous variable. Equation (2) is a conditional variance equation. $\omega$ is a constant term (mean), $\mu_{\mathrm{t}-1}{ }^{2}$ is a lag disturbance term (ARCH term) and is used to measure volatility information obtained from the previous period. $\mu_{\mathrm{t}-1}{ }^{2}$ is the conditional heteroscedasticity (GARCH term), which is the forecast variance of the previous period. The (p, q) in the GARCH (p, q) model refers to the GARCH term of the order p and the ARCH term of the order q.

\section{RMB Exchange Rate Band Selection and Data Processing}

This paper selects the USD/CNY exchange rate mid-day data from January 2, 2008 to December 29, 2017 (source: SAFE website). For ease of analysis, the logarithmic processing of the original data, the sequence was recorded as $l s_{t}$, the sequence $l s_{t}$ is obviously unstable, with a clear trend of 
fluctuations. For ADF test, the ADF value is -2.622588, which is greater than the critical value under the $5 \%$ confidence level. Therefore, it is determined that the $\mathrm{ls}_{\mathrm{t}}$ sequence has a unit root, and the sequence is not stable. In order to obtain a stable time series, the difference between $1 s_{t}$ sequences is ils $s_{t}=l s_{t}-l s_{t-1}$, and ils $s_{t}$ is the volatility of the exchange rate, as shown in Figure 1. From the data distribution point of view, the data is generally located near the value of 0 , and the RMB exchange rate fluctuations in both directions are significant. The fluctuation of the sequence has obvious clustering effect and there is heteroscedasticity.

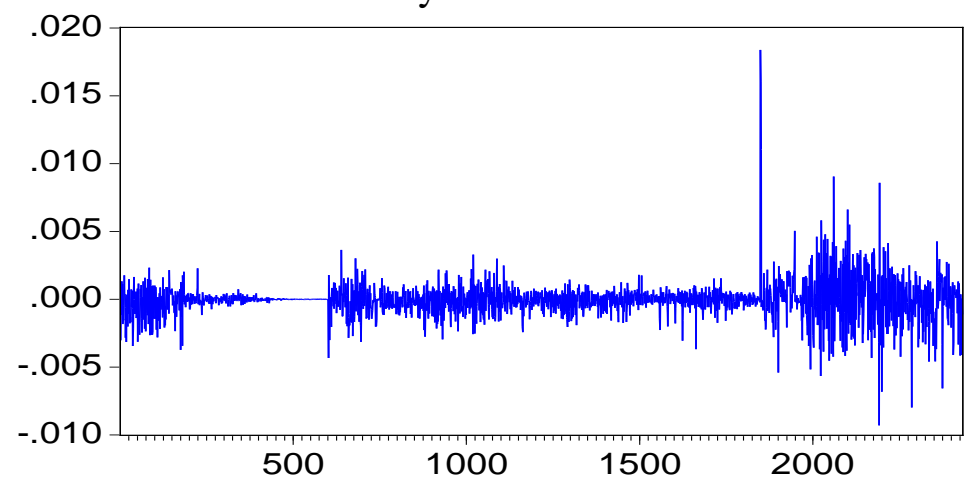

Fig.1

ADF test was performed on the ils $s_{t}$ sequence. The ADF value was -44.72545 , which was less than the critical value at the confidence level of $5 \%$. Therefore, there was no unit root in the ils $s_{t}$ sequence, and the sequence was stable. An autocorrelation test of the sequence revealed that the sequence has high-order autocorrelation and partial autocorrelation. Based on the above analysis, the differential exchange rate log series is stable, with heteroscedasticity and autocorrelation, and is applicable to the GARCH model.

Based on the fluctuations of the RMB exchange rate from 2008 to 2017 and the RMB exchange rate reform adjustment node, the RMB exchange rate sequence is divided into five phases, from January 2, 2008 to August 29, 2008, August 29, 2008 to June 21, 2010, June 21, 2010 to March 17, 2014, March 17, 2014 to August 11, 2015 and August 11, 2015 to December 29, 2017.

\section{Modeling, Forecasting and Analysis}

Based on the previous research experience and the test and analysis of the RMB exchange rate log-difference sequence, this paper establishes the $\operatorname{GARCH}(1,1)$ model to fit the exchange rate fluctuation series. For the mean model, since the first order autocorrelation coefficient and the partial autocorrelation coefficient are higher, the mean equation is established as ils $=\lambda$ ils $(-1)+c+\mu$. After regression, the following equation is obtained:

$$
\text { ils }=0.09551 * \operatorname{ILS}(-1)-3.73218 \mathrm{e}-05
$$

(AIC=-10.48149,SC=-10.47699)

Under the condition of first-order lag, $\mathrm{F}$ value of the $\mathrm{LM}$ test is 3.3734 , obs* $\mathrm{R}^{2}$ is 3.3730 , and the concomitant probabilities are 0.0664, 0.0663, respectively. The residual has ARCH effect. The GARCH $(1,1)$ model regression results are shown in Table 1.

Table 1. Regression Results

\begin{tabular}{c|c|c|c|c|c}
\hline \hline 1 & $0.3 \%$ & -0.02801 & $-3.8078 \mathrm{e}-04$ & 0.66479 & 0.00006 \\
\hline 2 & - & 0.07950 & $5.7473 \mathrm{e}-07$ & 0.91789 & 0.07734 \\
\hline 3 & $0.5 \%$ & 0.06632 & $9.3136 \mathrm{e}-05$ & 0.92328 & 0.05973 \\
\hline 4 & $2 \%$ & 0.08261 & $4.5335 \mathrm{e}-06$ & 0.48224 & 0.35306 \\
\hline 5 & $3 \%$ & 0.04807 & $7.4046 \mathrm{e}-05$ & 0.46879 & 0.27802 \\
\hline 1 & $0.3 \%$ & -0.02801 & $-3.8078 \mathrm{e}-04$ & 0.66479 & 0.00006 \\
\hline
\end{tabular}

The sums of ARCH terms and GARCH terms are both less than 1, and the model satisfies the parameter constraints. 
It can be seen from the above regression results that:

(1) When the amplitude limit is less than $1 \%$, the coefficient of conditional heteroscedasticity is greater than the conditional heteroscedasticity coefficient when the amplitude limit is greater than $1 \%$. When the amplitude limit is $0.5 \%$, the conditional heteroscedasticity coefficient is the largest, and then the conditional heteroscedasticity coefficient decreases as the amplitude limit increases. It shows that the relaxation of the volatility limit reduces the clustering effect of the US dollar against the RMB exchange rate, and the memory of the exchange rate series weakens.

(2) When the amplitude limit is less than $1 \%$, the coefficient of the random disturbance term is far less than the random disturbance factor coefficient when the amplitude limit is greater than $1 \%$. This shows that with the increase in the degree of exchange rate marketization, external stimuli have a longer impact on exchange rate sequences, and exchange rate sequences have increased sensitivity to events, which is more conducive to exchange rate stability and control.

\section{Recommendations on RMB Exchange Rate Reform Policy}

The empirical results of this paper show that the RMB exchange rate reform makes the exchange rate decision more market-oriented and has a significant role in maintaining exchange rate balance. Further promoting the marketization of the exchange rate system is an effective path for exchange rate reform. In response to the RMB exchange rate reform, the following policy recommendations are proposed:

(1) We will steadily push forward the reform of the RMB exchange rate and further study in-depth the range of reasonable exchange rate fluctuations. In the managed floating exchange rate system, excessive or too low volatility limits the normal fluctuations of the RMB exchange rate. The sensitivity of exchange rate fluctuations to external stimuli increases, and there is a strong memory. If the restrictions on the RMB volatility are relaxed to a larger level, this means that minor exchange rate fluctuations may be continuously amplified, thus affecting the domestic and international economic and financial environment and the stability of the system. As for the further relaxation of the RMB exchange rate volatility limit, it should be introduced during the period when the RMB exchange rate is relatively stable. This will not only effectively increase the marketization of the RMB exchange rate, but also minimize the sharp fluctuations in the exchange rate.

(2) Strengthen the flexibility of the RMB exchange rate and further adjust the formation mechanism of the RMB exchange rate. With the multi-polarization of the international economy and the improvement of the internationalization of the RMB, rational optimization of the RMB exchange rate pricing mechanism and assessment mechanism is a prerequisite for maintaining domestic and international economic balance and stability of the RMB exchange rate. Although the influence of the US dollar on the RMB exchange rate formation mechanism has gradually weakened, the weight of the basket currency still has some differences compared with the currency weight of the RMB exchange rate index. To further promote the marketization of the RMB exchange rate middle market can reduce the risk of reform and further relax the restrictions on the fluctuation of the RMB exchange rate.

\section{Acknowledgment}

This research project is supported by Science Foundation of Beijing Language and Culture University (supported by “the Fundamental Research Funds for the Central Universities”) (Approval number: 18YJ040001).

\section{References}

[1] Luo Wei, Wu Jianhong. Study on the Fluctuation of RMB Exchange Rate Based on GARCH Model, Mathematical Statistics and Management, 2009(2).

[2] Wang Jiani, Li Wenhao. Whether GARCH Model Can Provide Good Prediction of Volatility, 
Quantitative Economics and Technology Economics Research, 2005(6).

[3] Zhang Heng, Wu Ke. Research on the Dynamic Characteristics of RMB Exchange Rate Based on GARCH Model, Economic Mathematics, Vol.32 No.4, December 2015.

[4] Zhang Jian. Research on the Impact of RMB Exchange Rate Volatility Adjustment on RMB Exchange Rate Fluctuation Mechanism, Economics and Management Review, 2018, 2nd Issue. 